CYBERNETICS AND INFORMATION TECHNOLOGIES • Volume 20, No 6

Special Issue on New Developments in Scalable Computing

Sofia $\bullet 2020$

Print ISSN: 1311-9702; Online ISSN: 1314-4081

DOI: $10.2478 /$ cait-2020-0058

\title{
Two-Stage Search-Based Approach for Determining and Sorting of Mountain Hiking Routes Using Directed Weighted Multigraph
}

\author{
Zornitsa Dimitrova ${ }^{1}$, Vasil Dimitrov ${ }^{1}$, Daniela Borissova ${ }^{1}$, Ivan \\ Garvanov $^{2}$, Magdalena Garvanova ${ }^{2}$ \\ ${ }^{1}$ Institute of Information and Communication Technologies, Bulgarian Academy of Sciences, 1113 Sofia, \\ Bulgaria \\ ${ }^{2}$ University of Library Studies and Information Technologies, 1303 Sofia, Bulgaria \\ E-mails: zrn.dimitrova@gmail.com vasil.zdimitrov@gmail.com dborissova@iit.bas.bg \\ i.garvanov@unibit.bg m.garvanova@unibit.bg
}

Abstract: The mountain hiking destinations become more popular as this is one of the possible ways to cope with workplace stress and to prevent burnout. In contrast to the tourist destinations, mountain hiking requires special attention due to the variety of mountain trails satisfying the same starting and finishing point for a particular route. For the goal, a two-stage search-based approach for a determining of possible routes considering the users' preferences is developed. The first stage is focused on the determining of possible hiking routes taking into account the requirements and tourists' preferences, while the second stage concerns the sorting of already determined hiking routes. The applicability of the described approach is illustrated and the obtained results demonstrate the capability in searching and sorting of mountain hiking trails using directed weighted multigraph including tourists' preferences.

Keywords: Directed weighted multigraph, Search-based approach, Mountain hiking algorithm, Mountain routes sorting, Geographic scalability.

\section{Introduction}

The global market today is faced with diffident challenges including a variety of holiday aspects, tourism, and economics (P e trov [15]). Depending on the tourist destinations - world, cultural, holiday, religious, etc. - different parameters are to be taken into account in the determining of the most suitable one (Y ang et al. [22]; $\mathrm{Oh}$ [12]; Mutind a and May aka [10]). This concerns also the mountain hiking destinations that more often are used to escape hectic daily routine during the weekend or used as a holiday. The mountain hiking destinations become more popular as this is one of the possible ways to cope with workplace stress and to prevent burnout. The users face with huge amounts of information such as real-time news, blogs, advertising, promotions, etc., but only a small part of it is relevant to the 
interests of a particular user. For determining in which information is relevant to the user interests, the primary task of document categorization is to classify all nonrelevant documents from an incoming stream (X i a o et al. [21]).

With the increasing popularity of mountain tourism, the number of people that plan hikes without enough training, skills, and equipment also increases. The main reason for this trend is that not all tourists conscientiously perform the timeconsuming task of selecting and analysing mountain routes. The constant growth of Internet resources related to different vacation and tourist destinations requires some additional processing to refine the needed useful information to end-users. To cope with such kind of problems, the current article proposes an approach for a searching and sorting of mountain hiking trails using a directed weighted multigraph considering the tourists' preferences. For the goal, an algorithm for identifying possible routes considering the users' preferences is developed based on the information of a mountain represented as a multigraph. The obtained set of possible routes could be ranked additionally by specified criteria given by the users.

The rest of the article is organized as follows: Section 2 contains the literature review, Section 3 demonstrates the transformation of mountain hiking trails via a directed weighted multigraph, Section 4 describes the proposed two-stage searchbased approach for the determining and sorting of mountain hiking routes using weighted multigraph, Section 5 contains a case-study of numerical testing, Section 6 presents the results and discussions regarding the applicability of the proposed approach, while the conclusions and future investigations are drawn in Section 7.

\section{Literature review}

Most studies point out that the competitiveness of a tourist destination is a complex concept that has a strongly multi-dimensional nature related to different aspects of planning concerning journeys, mountain tourism, holidays, fishing, etc. People make different decisions from the preparation and planning of journeys to their return. To deal with such decision-making processes, some authors rely on utility theory where tourists maximize their satisfaction in making travel decisions by selecting the best alternative (S i r k a y a and W o od side [17]). Tourists prefer human interactions that give a sense of confidence about their trip and provide them an insight into the selected destination (W atf a and Sobh [20]). The destinations for tourism can be classified by their duration. In the short-term, it is possible to visit the destination almost immediately and do not require tourism infrastructure, while the long-term destinations should follow sustainable tourism practices and can be modelled by using the random utility model ( $\mathrm{Ka} \mathrm{s} \mathrm{k,} \mathrm{K} \mathrm{line}$ and L a mo ure u $\mathrm{x}$ [6]). The other possible approach to support the group holiday decision making process is realized by using of mixed-methods approach (Lan y un, Xub and Wagner [7]). Tabu search method could be combined with the concept of multi-attribute utility theory to determine the optimal tour when visiting several destinations in one trip ( $\mathrm{U}$ w a i s y, B a izala and Reditya [19]). Tour planning represents a challenging task for individuals visiting unfamiliar tourist destinations due to the availability of numerous variations in public transportation. A context-aware web application that derives 
personalized multimodal tours via selected urban attractions is proposed ( $\mathrm{G}$ a v a las et al. [5]). This application is based on a routing algorithm which is the core functionality of eCOMPASS. Also, some predictive models could be employed in open data analysis in the development of recommender systems to support tourists' decision making (P a n t a no et al. [13]; P a n t a n o, Priporas and S ty los [14]). The tourism industry could benefit from different intelligent digital recommender systems to assist a large number of online platforms in tourism industries ( $\mathrm{P}$ a $\mathrm{n} \mathrm{t}$ a $\mathrm{n}$ o et al. [13]).

The problem of searching for the most suited tourist destinations can be viewed as a problem of search-based software engineering involving multiple criteria. That is why many researchers are focused on finding more advanced techniques to cope with multi-objective optimization (Ramirez, Romero and Ventura [16]). This means the obtained solution will be dependable on particular user preferences. Consideration of the user preferences in applications is a fundamental problem of preference and search-based software engineering (Ferreira, Vergilio and de $\mathrm{S}$ ou z a [4]). The recent research demonstrates some hybrid search strategies that combine database searches with snowballing (Mourao et al. [8]). The main challenge in software analytic is how to understand unstructured data. The authors propose a combination of Latent Dirichlet allocation and search-based optimizer for finding related topics within an unstructured text ( $\mathrm{Agrawal}, \mathrm{Fu}$ and $\mathrm{Menzies}$ [1]). An alternative scheme of the architectural style can be viewed as graph grammar (Thong ku m and V a tan a w o od [18]). This is due to the specifics of graphs that are able to provide a universally adopted data structure to model component-based systems ( $\mathrm{Naddaf}$ and $\mathrm{Rafe}$ [11]). Furthermore, some patterns that express reusable knowledge can be empirically discovered and used in software architecture (A hmad et al. [2]). The existence of different algorithms for graph data and developed software system for visual processing of graph problems (M u s t a k e r o v [9]; B oris sova and Mustakerov [3]) motivate the authors to represent the tourists' items as directed weighted multigraph.

\section{Mountain hiking trails as directed weighted multigraph}

Determining of the most suitable mountain hiking trail depends on many parameters including the formed group of tourists but also involves some particular parameters of mountain hiking trails. For example, climbing and descending take different times and have different types of body stress, which are factors that have to be considered in the selection of a mountain hiking trail. All these parameters of the mountain are making it appropriate to be represented as a directed weighted multigraph. The tourist items (like peaks, hut, shelter, etc.) can be expressed by the vertices of the graph, while the routes between two adjacent objects could be represented by the directed graph edges. The hiking trails between the tourists' items should be considered as unidirectional edges of the graph because of the mountain hiking specifics - climbing in one direction is very different activities from descending on the other. A frequent situation is two or more hiking trails to connect two adjacent tourist items. In such a way, the variety of mountain hiking trails can be expressed by using some of their 
parameters that are important to the tourists, as weights of the corresponding directed multigraph's edges. There are also global parameters that are valid for the whole mountain or for large parts of it. Such parameters are the latitude and altitude of the mountain, which defines the duration of the seasons, the bright hours of the day, and others. Consequently, for each particular mountain, the network of tourist items and hiking trails could be expressed via directed weighted multigraph $G$ as follows:

(1)

$$
G(V, E, W(E)) \text {, }
$$

where $V=\left\{v_{1}, v_{2}, \ldots, v_{n}\right\}$ is the set of vertices representing the tourist items, $E=\left\{e_{1}, e_{2}, \ldots, e_{m}\right\}$ is the set of edges representing hiking trails connecting the tourist items, and $W(E)=\left\{w_{1}, w_{2}, \ldots, w_{l}\right\}$ expresses the set of edge's weights expressed as a function of different parameters of the hiking trails like distance, time duration, seasonality, etc.

Considering the advantages of the modern high-performance computing systems, there is no limit to the amount of data processed. To illustrate the described approach, a part of Rila Mountain with actual tourist items and possible hiking trails with the presence of hiking markings is used as shown in Fig. 1.

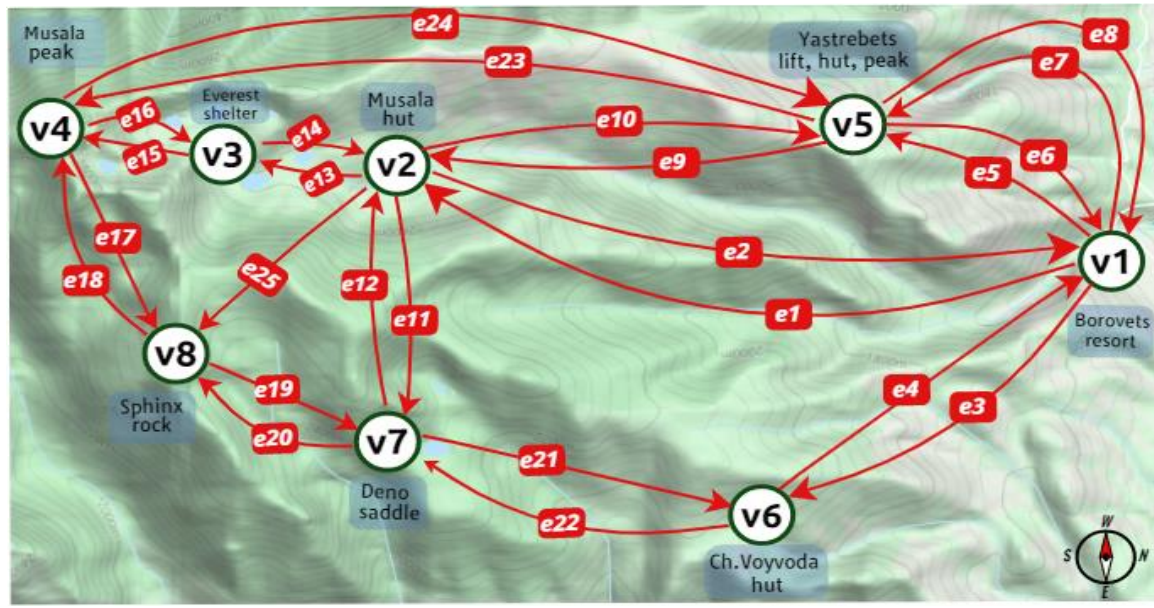

Fig. 1. Part of Rila Mountain with some tourist items and possible mountain hiking trails

This part of Rila Mountain is composed of 8 vertices (items) and 25 possible graph edges. The weighted components of graph edges represent the following characteristics of a mountain hiking trails are to be taken into account: (1) estimated average duration for crossing the hiking trail in hours; (2) length of the hiking trail in kilometres; (3) cumulative elevation gain (or total ascent); (4) cumulative elevation loss (or total descent); (5) season - in which seasons the hiking trail is safe to be crossed (winter, summer or both); (6) additional information which is not used by determining and sorting algorithms but is displayed to the user as description of the mountain hiking trails. This may include the type of marking, hiking trail type, and pavement, water sources availability (number and type), frequency of usage and existence of possible risks or dangers. The used parameters to express the weights of particular edges as a function of different parameters of the hiking trails from Fig. 1 are shown in Table 1. 
Table 1. Evaluation parameters of mountain hiking trails

\begin{tabular}{|c|c|c|c|c|c|}
\hline Edge & Duration & Length $(\mathrm{km})$ & Ascent $(\mathrm{m})$ & Descent $(\mathrm{m})$ & Season \\
\hline$e 1$ & $4 \mathrm{~h} 10 \mathrm{~min}$ & 8.500 & 1090 & 40 & all season \\
\hline$e 2$ & $3 \mathrm{~h} 30 \mathrm{~min}$ & 8.500 & 40 & 1090 & all season \\
\hline$e 4$ & $2 \mathrm{~h} 30 \mathrm{~min}$ & 7.500 & 605 & 20 & all season \\
\hline$e 5$ & $1 \mathrm{~h} 50 \mathrm{~min}$ & 7.500 & 20 & 605 & all season \\
\hline$e 6$ & $23 \mathrm{~min}$ & $0(4.800)$ & $0(1080)$ & 0 & all season \\
\hline$e 7$ & $23 \mathrm{~min}$ & $0(4.800)$ & 0 & $0(1080)$ & all season \\
\hline$e 8$ & $3 \mathrm{~h} 50 \mathrm{~min}$ & 7.300 & 1170 & 90 & all season \\
\hline$e 9$ & $3 \mathrm{~h} 10 \mathrm{~min}$ & 7.300 & 90 & 1170 & all season \\
\hline$e 10$ & $1 \mathrm{~h}$ & 3.500 & 40 & 20 & all season \\
\hline$e 11$ & $1 \mathrm{~h}$ & 3.500 & 20 & 40 & all season \\
\hline$e 12$ & $35 \mathrm{~min}$ & 1.700 & 160 & 0 & summer \\
\hline$e 13$ & $20 \mathrm{~min}$ & 1.700 & 0 & 160 & summer \\
\hline$e 14$ & $1 \mathrm{~h} 10 \mathrm{~min}$ & 2.500 & 320 & 0 & all season \\
\hline$e 15$ & $40 \mathrm{~min}$ & 2.500 & 0 & 320 & all season \\
\hline$e 16$ & $30 \mathrm{~min}$ & 0.700 & 0 & 216 & all season \\
\hline$e 17$ & $1 \mathrm{~h} 20 \mathrm{~min}$ & 2.400 & 110 & 307 & summer \\
\hline$e 18$ & $1 \mathrm{~h} 40 \mathrm{~min}$ & 2.400 & 307 & 110 & summer \\
\hline$e 19$ & $50 \mathrm{~min}$ & 1.800 & 90 & 250 & summer \\
\hline$e 20$ & $1 \mathrm{~h} 10 \mathrm{~min}$ & 1.800 & 250 & 90 & summer \\
\hline$e 21$ & $2 \mathrm{~h}$ & 5.500 & 20 & 570 & summer \\
\hline$e 22$ & $2 \mathrm{~h} 35 \mathrm{~min}$ & 5.500 & 570 & 20 & summer \\
\hline$e 23$ & $3 \mathrm{~h} 20 \mathrm{~min}$ & 6.200 & 607 & 150 & summer \\
\hline$e 24$ & $3 \mathrm{~h} 10 \mathrm{~min}$ & 6.200 & 150 & 607 & summer \\
\hline$e 25$ & $1 \mathrm{~h} 15 \mathrm{~min}$ & 2.000 & 300 & 0 & summer \\
\hline & & & & & \\
\hline
\end{tabular}

It should be noted that the length of the paths and elevation for edges $e 5$ and $e 6$ are equal to 0 as they represent a cable transport line (gondola lift) and this distance will not be walked by the tourist. When the edges between two nodes express a symmetric relation, the values for ascent and descent are swapped in both directions. This is due to cumulative elevation gain which refers to the sum of every gain in elevation throughout an entire hiking trail and transforms to cumulative elevation loss in the backward direction. The edge $e 25$ expresses situation as there is no equivalent in the opposite direction, due to the safety recommendations. Because of the high avalanche danger of the terrain during the winter season, the passing of some edges is forbidden. That is why the seasonality parameter for these edges has a value summer.

4. Two-stage search-based approach for determining and sorting of mountain hiking routes using weighted multigraph and different tourists' preferences

The proposed two-stage search-based approach for determining and sorting of mountain hiking trails relies on reliable input information concerning both mountain trails and tourists' preferences as shown in Fig. 2. 


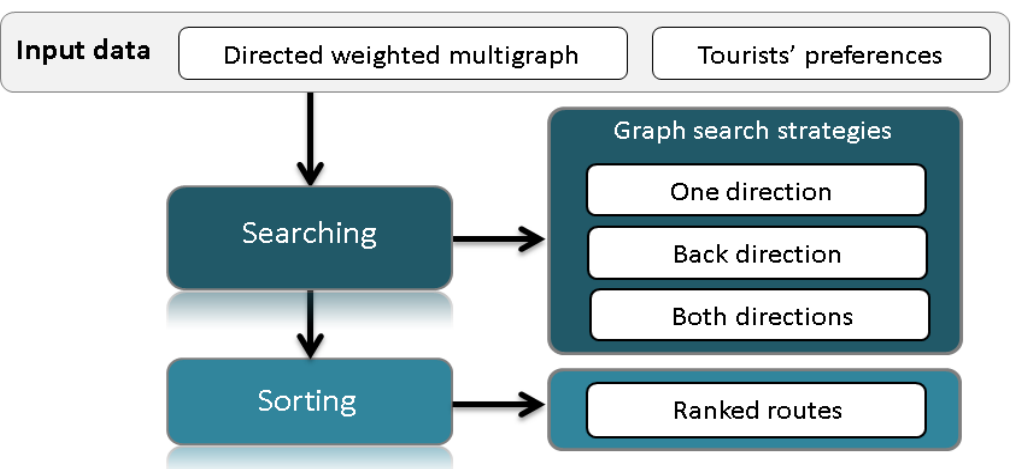

Fig. 2. A two-stage search-based approach for determining and sorting of mountain hiking routes using weighted multigraph

The essential part of the input information is related to the collection and processing of all required data about the mountain and transforming this data into a directed weighted multigraph. To facilitate the search within a directed multigraph, the relations and other components of the graph should be expressed by an adjacency list, an adjacency matrix, an incidence matrix, corresponding tables, etc. For example, the reviewed directed multigraph of the Rila Mountain, given by the adjacency matrix $G\left[v_{i}, v_{j}\right]$ is shown in Table 2 where the rows represent the initial vertices $v_{i}$ and the columns represent end vertices $v_{j}$.

Table 2. Adjacency matrix for particular excerpt of Rila Mountain

\begin{tabular}{|c|c|c|c|c|c|c|c|c|}
\hline Vertex & $v_{1}$ & $v_{2}$ & $v_{3}$ & $v_{4}$ & $v_{5}$ & $v_{6}$ & $v_{7}$ & $v_{8}$ \\
\hline$v_{1}$ & 0 & 1 & 0 & 0 & 2 & 1 & 0 & 0 \\
\hline$v_{2}$ & 1 & 0 & 1 & 0 & 1 & 0 & 1 & 1 \\
\hline$v_{3}$ & 0 & 1 & 0 & 1 & 0 & 0 & 0 & 0 \\
\hline$v_{4}$ & 0 & 0 & 1 & 0 & 1 & 0 & 0 & 1 \\
\hline$v_{5}$ & 2 & 1 & 0 & 1 & 0 & 0 & 0 & 0 \\
\hline$v_{6}$ & 1 & 0 & 0 & 0 & 0 & 0 & 1 & 0 \\
\hline$v_{7}$ & 0 & 1 & 0 & 0 & 0 & 1 & 0 & 1 \\
\hline$v_{8}$ & 0 & 0 & 0 & 1 & 0 & 0 & 1 & 0 \\
\hline
\end{tabular}

The next part of needed input information concerns the tourists' preferences including all parameters influencing the weight of each graph edges (possible mountain hiking trails). These parameters are used as restrictions in the next stage where an algorithm for determining of possible routes considering given tourists' preferences is proposed. The tourists' preferences include: (1) start point for the mountain route; (2) goal point of the route; (3) finish point of the route - in some cases, the starting and ending points could be the same but the routes in both directions could differ; (4) start and finish of a hiking trip, expressed by a calendar date/s that will give the information about the season; (5) the time for starting and ending the hike that will determine the maximum duration of mountain hike.

The first stage of the described approach aims to determine the possible routes considering given tourists' preferences. This is realized by the following proposed algorithm with six steps as shown in Fig. 3. 


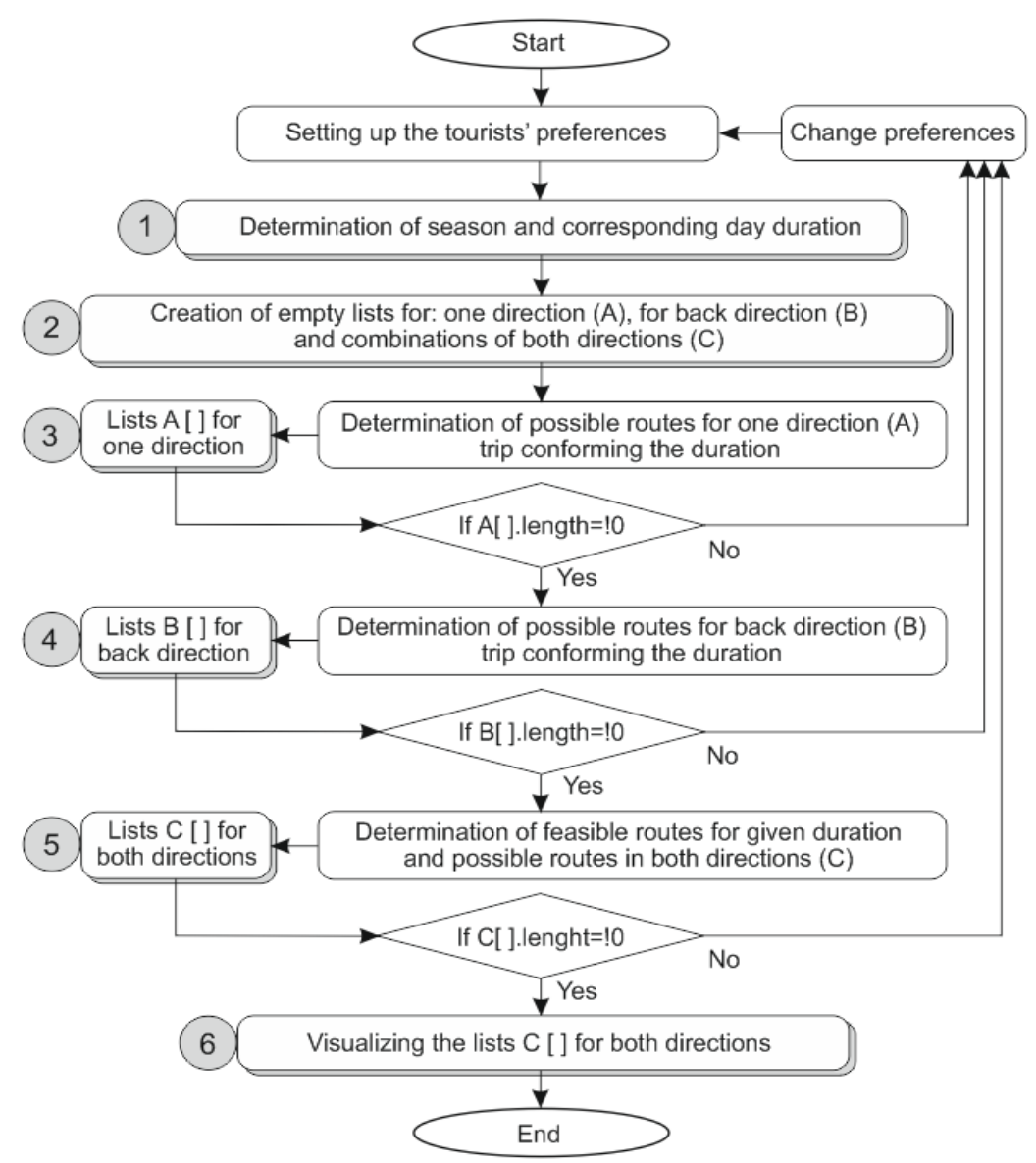

Fig. 3. Algorithm for determining of all possible routes taking into account the given tourists' preferences simultaneously

Step 1. The first step aims to extract the information about the season and respectively the daylight duration and maximum hike duration from the given tourists' preferences. The geographical location of the mountain and the dates of the hike are used to determine both the daylight duration and the different seasons, so the maximum estimated hiking time should be calculated taking into account the day duration for the chosen dates and the time for starting the hike.

Step 2. Three separate empty data structure are to be created: for one direction, for back direction and for the combination of both directions corresponding to the possible routes.

Step 3. Intermediate generation of the possible routes for one direction taking into account the input data about the selected starting vertex, goal vertex, season restriction and maximum hike duration. The graph search algorithm should satisfy the following conditions:

$$
\begin{gathered}
D_{\text {hike }} \geq D_{r 1}, \\
D_{r 1}=\sum_{i=0}^{n} D_{i},
\end{gathered}
$$


where $D_{\text {hike }}$ is the maximum hike duration route, $D_{r 1}$ is the route duration in one direction and is determined by the sum of edges $D_{i}$ involved in one direction.

Step 4. Intermediate generation of the possible routes for back direction. This step is identical to Step 3, but the intermediate routes are generated form the goal vertex to the end vertex, and

$$
\begin{gathered}
D_{\text {hike }} \geq D_{r 2}, \\
D_{r 2}=\sum_{j=0}^{n} D_{j},
\end{gathered}
$$

where $D_{\text {hike }}$ is the maximum hike duration route, $D_{r 2}$ is the route duration in back direction and is determined by the sum of edges $D_{j}$ involved in the back direction.

Step 5. The combinations of possible routes $D_{i, r 1}$ and $D_{j, r 2}$ have to comply with the following restriction:

$$
D_{\text {hike }} \leq D_{r 1}+D_{r 2} \text {. }
$$

If this list is empty, the tourist should change his preferences.

Step 6. On this step, proper visualization of the determined routes in both directions is to be done.

On the second stage of the proposed approach (Fig. 2), there is a possibility for a single criterion sorting of the list of routes determined from the previous stage. If there is only one route in list " $\mathrm{C}$ " it will be the only alternative the tourist can choose and this stage can be skipped. If this is not the case, the following sorting algorithm can be used as illustrated in Fig. 4.

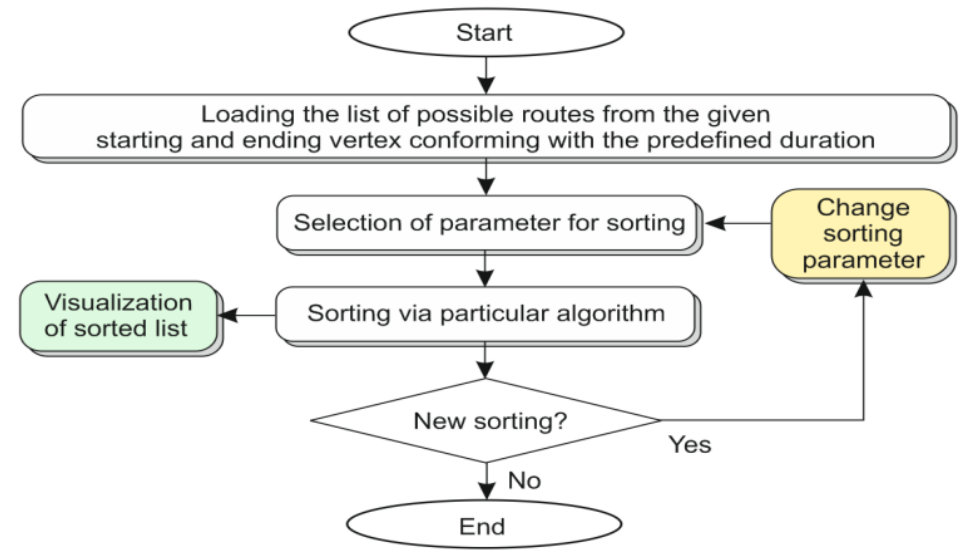

Fig. 4. Sorting of all determined routes taking into account the given tourists' preferences

The input data for the sorting algorithm are the list of determined routes and the possible sorting criteria. The routes can be ranked toward each criterion used as tourists' preferences except season (hike duration; length of the route; ascent; descent). To perform such sorting some well-known sorting algorithms can be used. The ranked list of routes can be arranged in increasing or decreasing order and then appropriately visualized. This process of sorting toward selected criterion can be repeated multiple times for all criteria according to tourists' preferences. Thus tourists will have all the information needed to make reasonable choices. 


\section{Numerical testing}

The proposed methodology and algorithms for determining and sorting of mountain hiking trails are demonstrated by using input data about a part of Rila Mountain as described in Table 1. Two cases with the same start and finish point are investigated to demonstrate the applicability of the proposed approach. The tourists' preferences for both cases start from Borovets resort (V1) via the Musala peak (V2) and back to Borovets resort (V1). The values of the tourists' preferences are shown in Table 3.

Table 3. Tourists' preferences for Case 1 and Case 2

\begin{tabular}{|c|c|c|c|c|c|c|c|}
\hline Case & Date start & Date finish & Time start & $\begin{array}{c}\text { Time } \\
\text { finish }\end{array}$ & Season & $\begin{array}{c}\text { Max hike } \\
\text { duration }\end{array}$ & $\begin{array}{c}\text { Sorting } \\
\text { criterion }\end{array}$ \\
\hline Case 1 & 22 February & 23 February & $13: 00$ & $13: 00$ & winter & $10 \mathrm{~h} \mathrm{50} \mathrm{min}$ & duration \\
\hline Case 2 & 5 July & 5 July & $10: 00$ & $17: 50$ & summer & $7 \mathrm{~h} 50 \mathrm{~min}$ & length \\
\hline
\end{tabular}

In Case 1, the hiking starts at 13:00 on 22 of February and should be finished at 13:00 on 23 of February. For these dates it is determined that for Rila Mountain the season is winter, the sunrise is at 7:15 and the sunset is at 18:05, which determine the maximum hike duration (up to $10 \mathrm{~h}$ and $50 \mathrm{~min}$ per day). In Case 2, the whole hike is planned for one day starting at 10:00 on 5 of July and finishes at 17:50, i.e., $7 \mathrm{~h}$ and $50 \mathrm{~min}$ is the maximum hike duration.

Using the tourists' preferences in Case 1, input data from Table 1 and the proposed algorithm, the possible routes are determined as shown in Table 4.

Table 4. Determined routes, sorted by hike duration in ascending order, by tourists' preferences

\begin{tabular}{|c|c|c|c|c|c|}
\hline No & Routes & Duration & Length $(\mathrm{km})$ & Ascent $(\mathrm{m})$ & Descent $(\mathrm{m})$ \\
\hline R1 & e5-e9-e13-e15-e16-e14-e10-e6 & $7 \mathrm{~h} 46 \mathrm{~min}$ & 13.400 & 596 & 596 \\
\hline $\mathrm{R} 2$ & e5-e9-e13-e15-e16-e14-e2 & 9 h 53 min & 18.400 & 616 & 1646 \\
\hline R3 & e5-e9-e13-e15-e16-e14-e10-e8 & $10 \mathrm{~h} 33 \mathrm{~min}$ & 20.700 & 686 & 1766 \\
\hline R4 & e1-e13-e15-e16-e14-e10-e6 & $10 \mathrm{~h} 33 \mathrm{~min}$ & 18.400 & 1646 & 616 \\
\hline
\end{tabular}

The obtained results in Case 2 based on the input data from Table 1 and using the proposed approach and sorting by a criterion of route length are shown in Table 5.

Table 5. Determined routes, sorted by length in descending order, by tourists' preferences

\begin{tabular}{|c|c|c|c|c|c|}
\hline No & Routes & Duration & Length $(\mathrm{km})$ & Ascent $(\mathrm{m})$ & Descent $(\mathrm{m})$ \\
\hline R1 & $e 5-e 23-e 17-e 19-e 12-e 10-e 6$ & $7 \mathrm{~h} 36 \mathrm{~min}$ & 15.600 & 827 & 907 \\
\hline R2 & $e 5-e 9-e 13-e 19-e 14-e 10-e 6$ & $7 \mathrm{~h} 46 \mathrm{~min}$ & 13.400 & 596 & 596 \\
\hline R3 & $e 5-e 9-e 13-e 15-e 24-e 6$ & $7 \mathrm{~h} 46 \mathrm{~min}$ & 12.900 & 726 & 627 \\
\hline R4 & $e 5-e 23-e 16-e 14-e 10-e 6$ & $7 \mathrm{~h} 16 \mathrm{~min}$ & 12.900 & 627 & 726 \\
\hline R5 & $e 5-e 23-e 24-e 6$ & $7 \mathrm{~h} 16 \mathrm{~min}$ & $12, .400$ & 757 & 757 \\
\hline
\end{tabular}




\section{Results and discussions}

The obtained results in both cases using the same start and finish point in different seasons determine different possible routes as illustrated in Fig 5.

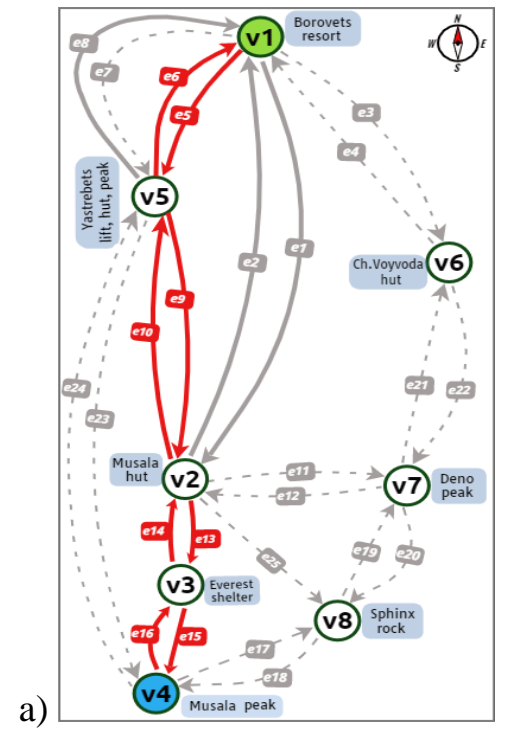

b)

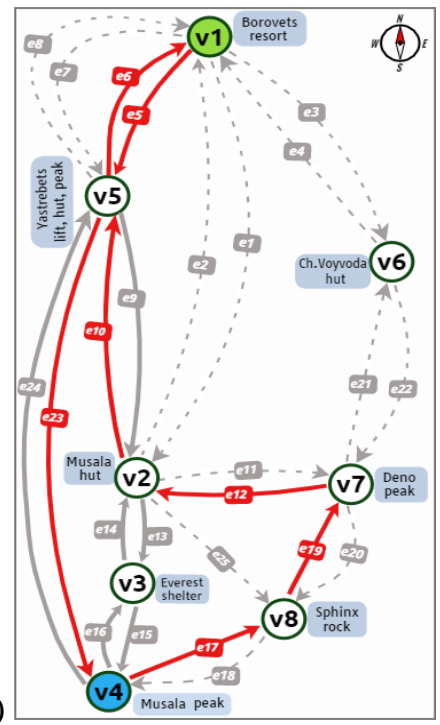

Fig. 5. Determined routes for two types of tourists' preferences: a) Case 1; b) Case 2

The routes that meet the tourist's preference toward hike dates, duration and ranking are shown in red. It is interesting to note, that hike in planning in winter, some of the routes satisfying the condition of maximum hike duration as route e5-e23-e24-e6 do not take place into the intermediate lists (Steps 3 and 4 of the algorithm) because they contain edges suitable only for passing in summer (Fig. 5a). The route with minimum duration in Case 1 passes through edges e5-e9-e13-e15e16-e14-e10-e6, which overall duration time is equal to $7 \mathrm{~h}$ and $46 \mathrm{~min}$. When the season has changed the set of possible routes has changed too (Fig. 5b). Despite the same start and finish point, the season influences the maximum daylight that reflects in the possible duration of a hike within a day. As the Case 2 concern one day hike, the most suitable sorting of routes is done in respect of route length (Fig. 5b).

In both cases, the distance and elevation, which are passed by a cable transport line at the edges $e 5$ and $e 6$, are excluded from the total length respectively elevation of the route containing these edges, but time is taken into account.

The concept of three key vertices and dividing the search for a route into two separate searches is close to the approach that the tourists use to plan their hikes in general. This allows the planning of both circular routes that start and finishes in one place and routes that cross the mountain. The conducted testing proved the applicability for generating alternative routes and subsequent sorting taking into account the tourist preferences. In addition, the use of a multigraph makes it possible and easy to expand the multigraph by adding additional nodes and arcs. This feature contributes to obtaining highly efficient solutions based on the proposed scalable 
algorithms for searching and sorting that are able to keep the same efficiency when the workload grows. All of this proves the scalability of the described approach by providing the ability to maintain effectiveness when expanding the weighted multigraph.

\section{Conclusions}

Mountain tourism can benefit from different intelligent systems that can be tailored to provide the information and assist the tourists in reasonable decision making. For this purpose an approach is proposed for determining and sorting of mountain hiking trails. The advantage of the described approach is the fact, that all relevant information about the mountain and available trails can be expressed by a directed weighted multigraph. The proposed scalable search-based algorithm realizes determining of the possible hiking routes considering different tourists' preferences. Once the possible routes are determined in accordance with the given preferences, another algorithm is invoked to rank these routes with respect to the most important criterion from a particular user point of view.

The proposed approach for determining and sorting of mountain hiking routes based on a weighted multigraph can be extended with the capability for hikes planning of several days' duration. This requires to introduce daily goal destination and finding routes that pass through a place appropriate for overnight stay (hut, shelter, camping) at the end of each day. Another direction for improvement is the possibility for the determining of routes between destinations in different mountains by connecting the individual graphs representing the mountain in one big graph, which is possible due to the scalability of the proposed algorithm.

Acknowledgments: This research is supported by the Project BG05M2OP001-1.001-0003 entitled "Center of Excellence in Informatics and Information and Communication Technologies".

\section{References}

1. A graw a l, A., W. F u, T. M en zi e s. What is Wrong with Topic Modeling? And How to Fix it Using Search-Based Software Engineering. - Information and Software Technology, Vol. 98, 2018, pp. 74-88.

2. A h mad, A., C. Pahl, A. B. A $1 \mathrm{ta} m i \mathrm{mi}$, A. A $1 \mathrm{r} e \mathrm{sh}$ id i. Mining Patterns from Change Logs to Support Reuse-Driven Evolution of Software Architectures. - Journal of Computer Science and Technology, Vol. 33, 2018, No 6, pp. 1278-1306.

3. Boris sova, D., I. Mustakerov. e-Learning Tool for Visualization of Shortest Paths Algorithms. - Trends Journal of Sciences Research, Vol. 2, 2015, No 3, pp. 84-89.

4. F e rr e i r a, T. N., S. R. V e r g i l i o, J. T e i x e i r a de $\mathrm{S}$ o u z a. Incorporating User Preferences in Search-Based Software Engineering: A Systematic Mapping Study. - Information and Software Technology, Vol. 90, 2017, pp. 55-69.

5. Gavalas, D., V. Kas apakis, C. Konstantopoulos, G. Pantziou, N. Vathis, C. Z a r o li a g i s. The eCOMPASS Multimodal Tourist Tour Planner. - Expert Systems with Applications, Vol. 42, 2015, pp. 7303-7316.

6. K a s k, S., C. K li n e, K. L a m o u r e u x. Modeling Tourist and Community Decision Making: The SAVE Market. - Annals of Tourism Research, Vol. 38, 2011, No 4, pp. 1387-1409. 
7. L a n y u n, Z., S. X u b, C. W a g n e r. Exploring the Group Holiday Decision-Making Process with the Support of Technology. - Information Processing and Management, Vol. 56, 2019, pp. 1409-1424.

8. Mourao, E., J.F.Pi mente l, L. Murta, M. Kalinowski, E. Mendes, C. Wohlin. On the Performance of Hybrid Search Strategies for Systematic Literature Reviews in Software Engineering. - Information and Software Technology, Vol. 123, 2020, 106294.

9. Must a k e rov, I. A Software System for Visual Processing of Graph Data. - Cybernetics and Information Technologies, Vol. 5, 2005, No 2, pp. 156-163.

10. Mutinda, R., M. M a y k a. Application of Destination Choice Model: Factors Influencing Domestic Tourists Destination Choice among Residents of Nairobi, Kenya. - Tourism Management, Vol. 3, 2012, pp. 1593-1597.

11. N add af, M. R., V. R a fe. Performance Modeling and Analysis of Software Architectures Specified through Graph Transformations. - Computing and Informatics, Vol. 32, 2013, pp. 797-826.

12. O h, C.-O. Incorporating Simplified Decision Rules into Tourist Decision Making Processes: A Case of Fishing Trips. - Ocean \& Coastal Management, Vol. 71, 2013, pp. 79-87.

13. Pantano, E., C.-V. Priporas, N. S tylos, C. Den nis. Facilitating Tourists' Decision Making through Open Data Analyses: A Novel Recommender System. - Tourism Management Perspectives, Vol. 31, 2019, pp. 323-331.

14. P a n t a n o, E., C.-V. Pri p or a s, N. S t y lo s. "You Will Like It!" Using Open Data to Predict Tourists' Response to a Tourist Attraction. - Tourism Management, Vol. 60, 2017, pp. 430-438.

15. P e tro v, I. Improving the Methodology of Market Structures Analysis with Innovative Concepts for Phase-Structure States and Set Concentration Index. - Economic Alternative, Vol. 1, 2016, pp. 5-14.

16. R a mir e z, A., J. R. R o m e r o, S. V e n t u r a. A Survey of Many-Objective Optimisation in Search-Based Software Engineering. - The Journal of Systems and Software, Vol. 149, 2019, pp. 382-395.

17. S i r a k a ya, E., A. G. Woods ide. Building and Testing Theories of Decision Making by Travellers. - Tourism Management, Vol. 26, 2005, pp. 815-832.

18. Th on g k u m, S., W. V a t a n a w o od. An Approach of Software Architectural Styles Detection Using Graph Grammar. - IACSIT International Journal of Engineering and Technology, Vol. 6, 2014, No 2, pp. 123-127.

19. U w a i s y, A. M., Z. K. A. B a i z a 1 a, M. Y. R e d i t y a. Recommendation of Scheduling Tourism Routes Using Tabu Search Method (Case Study Bandung). - Procedia Computer Science, Vol. 157, 2019, pp. 150-159.

20. W atfa, M. K., D. S o bh. Generative Smart Tourism, the Road for Big Data. - Journal of Advanced Management Science, Vol. 5, 2017, No 6, pp. 424-439.

21. X i a o, Y., B. L i u, J. Y i n, Z. H a o. A Multiple-Instance Stream Learning Framework for Adaptive Document Categorization. - Knowledge-Based Systems, Vol. 120, 2017, pp. 198-210.

22. Y a n g, X., K. Hung, W.-J. Hu ang, Y.-P. T s e n g. Tourism Representation by DMOs at Religious Sites: A Case of Shaolin Temple, China. - Tourism Management, Vol. 75, 2019, pp. 569-581.

Received: 25.07.2020; Second Version: 15.09.2020; Accepted: 25.09.2020 